\title{
Optimal Scheduling over Fading Broadcast Channels with an Energy Harvesting Transmitter
}

\author{
Omur Ozel $^{1}$, Jing Yang ${ }^{2}$, and Sennur Ulukus ${ }^{1}$ \\ ${ }^{1}$ Department of Electrical and Computer Engineering, University of Maryland, College Park, MD 20742 \\ ${ }^{2}$ Department of Electrical and Computer Engineering, University of Wisconsin-Madison, Madison, WI 53706
}

\begin{abstract}
We consider an energy harvesting transmitter sending messages to two users over a fading AWGN broadcast channel. Energy required for communication arrives (is harvested) at the transmitter and a finite capacity battery stores it before being consumed for transmission. Based on a deterministic energy arrival and fading model, we obtain the maximum departure region in a given interval. We obtain the transmission policies that achieve the boundary of the maximum departure region by a directional waterfilling algorithm.
\end{abstract}

\section{INTRODUCTION}

A distinct characteristic of energy harvesting communication systems is that the energy required for communication arrives during the session in which communication takes place. The transmitter is able to harvest energy from nature in order to recharge its battery. The energy is modeled to arrive (be harvested) at random times and in random amounts. Therefore, transmission schemes must adapt to the incoming energy. In this paper, we consider communication with an energy harvesting transmitter over a fading AWGN broadcast channel.

In particular, we consider an energy harvesting transmitter that sends data to two receivers over a fading broadcast channel as in Fig. 1. Data for the two receivers are backlogged at the transmitter buffers while arriving energy is stored in a finite capacity battery. Optimally scheduling the transmissions is challenging: First, the channel fade levels vary in time and an opportunism is required to take advantage of good channel states. Second, the transmitter has to adapt its transmission power with respect to the available energy and also avoid possible energy overflows from the finite-capacity battery.

There has been recent research effort on understanding data transmission with an energy harvesting transmitter that has a rechargeable battery [1]-[7]. In [1], data transmission with energy harvesting sensors is considered, and the optimal online policy for controlling admissions into the data buffer is derived using a dynamic programming framework. In [2], energy management policies which stabilize the data queue are proposed for single-user communication and under a linear approximation, some delay optimality properties are derived. In [3], throughput optimal energy allocation is studied for energy harvesting systems in a time constrained slotted setting. In [4], [5], minimization of the transmission completion time is considered in an energy harvesting system and the optimal

This work was supported by NSF Grants CCF 07-29127, CNS 09-64632, CCF 09-64645 and CCF 10-18185.

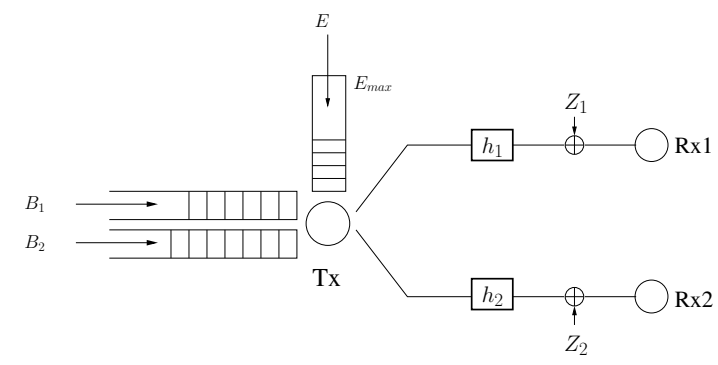

Fig. 1. The two-user fading broadcast channel with an energy harvesting transmitter.

solution is obtained using a geometric framework. In [6], energy harvesting transmitters with batteries of finite energy storage capacity are considered and the problem of throughput maximization by a deadline is solved in a static channel. In [7], offline and online optimal transmission policies are provided for a single-user energy harvesting transmitter operating in a fading channel. In [8]-[10] optimal offline policies are found for the static AWGN broadcast channel.

In this paper, we extend the line of research in offline optimal scheduling to the fading broadcast channel. We obtain the full characterization of the maximum departure region [8], [10] by a deadline $T$ and determine the optimal offline transmission policies that achieve the boundary of the maximum departure region. We show that the energy allocated in each epoch is determined by a directional waterfilling algorithm [7]. In particular, water level in between two energy arrivals is calculated by using the waterfilling scheme described in [11], [12]. If the water level is higher on the right, no energy is transfered; otherwise some energy is transferred to the future. Using the resulting water levels, portions of the power allocated for each user are found in each epoch separately.

\section{Two-User Fading Gaussian Broadcast Channel}

In two-user energy harvesting fading broadcast channels, the transmitter has three queues as in Fig. 1: two data queues where data packets for the two receivers are stored, and an energy queue where the arriving (harvested) energy is stored. The energy queue, i.e., the battery, can store at most $E_{\max }$ units of energy, which is used for transmission only, i.e., energy required for processing is not considered.

The received signals at the two receivers are

$$
Y_{1}=\sqrt{h_{1}} X+Z_{1}, \quad Y_{2}=\sqrt{h_{2}} X+Z_{2}
$$


where $X$ is the transmit signal, and $Z_{1}, Z_{2}$ are Gaussian noises with zero-mean and variances $\sigma_{1}^{2}$ and $\sigma_{2}^{2}$, respectively, and $h_{1}, h_{2}$ are the (squared) fading coefficients. As in [11], we reflect the effect of fading on the noise power. In particular, we obtain an equivalent broadcast channel by letting $n_{1}=\frac{\sigma_{1}^{2}}{h_{1}}$ and $n_{2}=\frac{\sigma_{2}^{2}}{h_{2}}$. If the channel fade levels are constant at $h_{1}$, $h_{2}$, the resulting broadcast channel capacity region $\mathcal{C}(P)$ has the following well-known characterization [13]:

$$
\begin{aligned}
& R_{1} \leq \frac{1}{2} \log \left(1+\frac{\alpha P}{(1-\alpha) P \mathbf{1}\left(n_{1}>n_{2}\right)+n_{1}}\right) \\
& R_{2} \leq \frac{1}{2} \log \left(1+\frac{(1-\alpha) P}{\alpha P \mathbf{1}\left(n_{2}>n_{1}\right)+n_{2}}\right)
\end{aligned}
$$

where $P$ is the average power available to the transmitter and $\alpha$ is the power share of user 1 . We call the receiver which observes smaller noise power as the stronger receiver. Note that changes in the fading levels of the channels cause time variations in the strength order of the receivers.

Whenever an input signal $x$ is transmitted with power $p$ in an epoch of duration $\ell$ in which the channel fades are constant at the levels $h_{1}$ and $h_{2}, R_{1} \ell$ and $R_{2} \ell$ bits of data are served out from the backlogs of receivers 1 and 2, with the cost of $p \ell$ units of energy depletion from the energy queue. Here, $\left(R_{1}, R_{2}\right)$ is the rate allocation for this epoch. $\left(R_{1}, R_{2}\right)$ must reside in the capacity region of the two-user AWGN broadcast channel $\mathcal{C}_{n_{1}, n_{2}}(p)$, indexed by the noise variances $n_{1}$ and $n_{2}$, which vary during the communication session. Extending this for continuous time, if at time $t$ the transmit power is $p(t)$ and the noise variances are $n_{1}(t)=\sigma_{1}^{2} / h_{1}(t)$ and $n_{2}(t)=\sigma_{2}^{2} / h_{2}(t)$, the instantaneous rate pairs satisfy $\left(R_{1}(t), R_{2}(t)\right) \in \mathcal{C}_{n_{1}(t), n_{2}(t)}(p(t))$. The transmission policy in a broadcast channel is comprised of the total power $p(t)$ and the portion of the total transmit power $\alpha(t)$ that is allocated for user 1 .

We consider an offline setting where the changes that occur in the energy and fading levels throughout the communication session are known by the transmitter a priori. The fading and energy levels change at discrete time instants $t_{1}^{f}, t_{2}^{f}, \ldots, t_{n}^{f}, \ldots$ and $t_{1}^{e}, t_{2}^{e}, \ldots, t_{n}^{e}, \ldots$, respectively. $N$ is the number of energy arrivals and $K$ is the number of changes in the joint fading level during the $[0, T]$ interval. Note that a change in the fading level means any change in the joint fading state $\left(h_{1}, h_{2}\right)$. We define an epoch as a time interval in which no energy arrival or channel fade level change occurs. We extend the definition of energy arrival sequence for the time instants at which a fading change occurs. In particular, the input energy for epoch $i$ is denoted as $E_{i-1}$ and it is equal to the amount of incoming energy if the epoch starts with an energy arrival; if epoch $i$ starts with a variation in the fading level without an energy arrival, $E_{i-1}=0$. Finally, we let $\ell_{i}$ be the length of the $i$ th epoch and $\left\{\left(n_{1 i}, n_{2 i}\right)\right\}_{i=1}^{N+K+1}$ be the sequence of noise variances of the equivalent broadcast channels.

There are two constraints on $p(t)$, due to energy arrivals at random times and also due to finite battery storage capacity. Let $h_{-}(t)=\max \left\{i: t_{i}^{e}<t\right\}$. Since energy that has not arrived yet cannot be used at the current time, there is a causality constraint:

$$
\int_{0}^{t} p(\tau) d \tau \leq \sum_{j=0}^{h_{-}(t)} E_{j}, \quad \forall t \in[0, T]
$$

Moreover, due to the finite battery storage capacity, if the energy level in the battery exceeds $E_{\max }$, some energy is lost due to overflow. In order to guarantee no-energy-overflow, it suffices to ensure that the energy level in the battery never exceeds $E_{\max }$ at the times of energy arrivals. Hence, we have the following no-energy-overflow constraint:

$$
\int_{0}^{t} p(\tau) d \tau \geq\left(\sum_{i=0}^{h_{+}(t)} E_{i}-E_{\max }\right)^{+}, \quad \forall t \in[0, T]
$$

where $(x)^{+}=\max (0, x)$ and $h_{+}(t)=\max \left\{i: t_{i}^{e} \leq t\right\}$.

\section{Maximum Departure Region}

In this section, we characterize the trade-off between the performances of user 1 and user 2 by finding the region of bits sent for receivers 1 and 2 in the interval $[0, T]$ with offline knowledge of energy and fading variations. The number of bits sent for receivers 1 and 2 are:

$$
B_{1}=\int_{0}^{T} R_{1}(\tau) d \tau, \quad B_{2}=\int_{0}^{T} R_{2}(\tau) d \tau
$$

The instantaneous rates $R_{1}(t)$ and $R_{2}(t)$ are determined as a function of the instantaneous power policy $p(t)$ with portion $\alpha(t)$ via (2) and (3).

Definition 1 For any fixed transmission duration $T$, the maximum departure region, denoted as $\mathcal{D}(T)$, is the union of $\mathcal{R}\left(B_{1}, B_{2}\right)=\left\{\left(b_{1}, b_{2}\right): 0 \leq b_{1} \leq B_{1}, 0 \leq b_{2} \leq B_{2}\right\}$ where $\left(B_{1}, B_{2}\right)$ is the total number of bits sent by some power allocation policy that satisfies energy causality (4) and noenergy-overflow (5) conditions over the duration $[0, T)$.

We have the following lemma, the proof of which can be carried out following the proofs of Lemma 2 in [8] and Lemma 1 in [10] and hence is skipped here for brevity.

\section{Lemma $1 \mathcal{D}(T)$ is a convex region.}

In the following analysis, we call any policy that satisfies energy causality and no-energy-overflow conditions feasible. We call a feasible policy optimal if it achieves the boundary of $\mathcal{D}(T)$. We first note that due to the convexity of $\mathcal{D}(T)$ in Lemma 1 and the concave power-rate relation, an optimal policy should remain constant in any epoch (c.f. Lemma 1 in [8] and Lemma 2 in [4], [5]). Therefore, the policy is a sequence of powers $\left\{p_{i}\right\}_{i=1}^{N+K+1}$ and user 1's power share $\left\{\alpha_{i}\right\}_{i=1}^{N+K+1}$. The causality and no-energy-overflow conditions 
reduce to the following constraints, respectively:

$$
\begin{aligned}
& \sum_{i=1}^{k} p_{i} \ell_{i} \leq \sum_{i=0}^{k-1} E_{i}, \quad k=1, \ldots, N+K+1 \\
& \sum_{i=1}^{k} p_{i} \ell_{i} \geq\left(\sum_{i=0}^{k} E_{i}-E_{\text {max }}\right)^{+}, \quad k=1, \ldots, N+K
\end{aligned}
$$

We define the following functions:

$$
\begin{aligned}
& r_{1}\left(n_{1}, n_{2}, \alpha, p\right)=\frac{1}{2} \log _{2}\left(1+\frac{\alpha p}{(1-\alpha) p \mathbf{1}\left(n_{1}>n_{2}\right)+n_{1}}\right) \\
& r_{2}\left(n_{1}, n_{2}, \alpha, p\right)=\frac{1}{2} \log _{2}\left(1+\frac{(1-\alpha) p}{\alpha p \mathbf{1}\left(n_{2}>n_{1}\right)+n_{2}}\right)
\end{aligned}
$$

By Lemma 1, any point on the boundary of $\mathcal{D}(T)$ can be characterized by solving the following optimization problem:

$$
\begin{aligned}
\max _{\mathbf{p}, \boldsymbol{\alpha}} & \mu_{1} \sum_{i=1}^{N+K+1} r_{1}\left(n_{1 i}, n_{2 i}, \alpha_{i}, p_{i}\right) \ell_{i}+\mu_{2} \sum_{i=1}^{N+K+1} r_{2}\left(n_{1 i}, n_{2 i}, \alpha_{i}, p_{i}\right) \ell_{i} \\
\text { s.t. } & \sum_{i=1}^{k} p_{i} \ell_{i} \leq \sum_{i=0}^{k-1} E_{i}, \quad \forall k \\
& \sum_{i=1}^{k} p_{i} \ell_{i} \geq\left(\sum_{i=0}^{k} E_{i}-E_{\text {max }}\right)^{+}, \quad \forall k
\end{aligned}
$$

In (11), $\mathbf{p}, \boldsymbol{\alpha}$ denote the vector of total powers and the power shares, respectively. (11) is not a convex problem as the variables $p_{i}, \alpha_{i}$ appear in a product form in the expression of $r_{i}\left(n_{1}, n_{2}, \alpha, p\right)$, causing it to be non-concave in $\alpha_{i}$ and $p_{i}$. However, $\mu_{1} r_{1}\left(n_{1}, n_{2}, \alpha, p\right)+\mu_{2} r_{2}\left(n_{1}, n_{2}, \alpha, p\right)$ is concave with respect to $p$ for any given $n_{1}, n_{2}, \alpha$. Using this property, we solve (11) in two steps as in [10]. We optimize over $\alpha_{i}$ first and then over the total power $p_{i}$. Consider the single variable optimization problem:

$$
\max _{0 \leq \alpha \leq 1} \mu_{1} r_{1}\left(n_{1}, n_{2}, \alpha, p\right)+\mu_{2} r_{2}\left(n_{1}, n_{2}, \alpha, p\right)
$$

The problem in (12) is solved at $\alpha=\alpha^{*}\left(n_{1}, n_{2}, p\right)$. Assume $n_{1}<n_{2}$ and let $\mu=\mu_{2} / \mu_{1}$ and $\sigma^{2}=\frac{n_{2}}{n_{1}}$. We have shown in [10] that if $1<\mu<\sigma^{2}, \alpha^{*}\left(n_{1}, n_{2}, p\right)$ is expressed as:

$$
\alpha^{*}\left(n_{1}, n_{2}, p\right)= \begin{cases}1, & 0 \leq p \leq \frac{\mu_{2} n_{1}-\mu_{1} n_{2}}{\mu_{1}-\mu_{2}} \\ \frac{1}{p} \frac{\mu_{2} n_{1}-\mu_{1} n_{2}}{\mu_{1}-\mu_{2}}, & p \geq \frac{\mu_{2} n_{1}-\mu_{1} n_{2}}{\mu_{1}-\mu_{2}}\end{cases}
$$

In the extreme cases, $\alpha^{*}\left(n_{1}, n_{2}, p\right)=1$ if $\mu \leq 1$ and $\alpha^{*}\left(n_{1}, n_{2}, p\right)=0$ if $\mu \geq \sigma^{2}$. If the order of noises is the other way, i.e., $n_{2}<n_{1}$, by changing the definitions as $\mu=\frac{\mu_{1}}{\mu_{2}}$ and $\sigma^{2}=\frac{n_{1}}{n_{2}}$, for $\alpha^{*}$ in (13), $1-\alpha^{*}\left(n_{1}, n_{2}, p\right)$ gives the solution of (12). Denoting the solution in (13) as $\alpha^{*}$ in short, define

$$
\begin{aligned}
& r_{1}^{*}\left(n_{1}, n_{2}, p\right) \triangleq \frac{1}{2} \log _{2}\left(1+\frac{\alpha^{*} p}{\left(1-\alpha^{*}\right) p \mathbf{1}\left(n_{1}>n_{2}\right)+n_{1}}\right) \\
& r_{2}^{*}\left(n_{1}, n_{2}, p\right) \triangleq \frac{1}{2} \log _{2}\left(1+\frac{\left(1-\alpha^{*}\right) p}{\alpha^{*} p \mathbf{1}\left(n_{2}>n_{1}\right)+n_{2}}\right)
\end{aligned}
$$

We define

$$
g\left(n_{1}, n_{2}, p\right)=\mu_{1} r_{1}^{*}\left(n_{1}, n_{2}, p\right)+\mu_{2} r_{2}^{*}\left(n_{1}, n_{2}, p\right)
$$

We have shown in [10] that $g\left(n_{1}, n_{2}, p\right)$ is a strictly concave function of $p$. In particular, it has a continuous monotone decreasing first derivative: for $n_{1}<n_{2}$, whenever $\alpha^{*}\left(n_{1}, n_{2}, p\right)=1$, the derivative is $\frac{\mu_{1}}{p+n_{1}}$ and otherwise, it is $\frac{\mu_{2}}{p+n_{2}}$. Similarly, if $n_{2}<n_{1}$, whenever $\alpha^{*}\left(n_{1}, n_{2}, p\right)=0$, the derivative is $\frac{\mu_{2}}{p+n_{2}}$ and otherwise, it is $\frac{\mu_{1}}{p+n_{1}}$. Hence, by first optimizing over $\alpha_{i}$ in (11), we obtain the following convex optimization problem over the total power sequence $\left\{p_{i}\right\}$.

$$
\begin{aligned}
\max _{\mathbf{p}} & \sum_{i=1}^{N+K+1} g\left(n_{1 i}, n_{2 i}, p_{i}\right) \ell_{i} \\
\text { s.t. } & \sum_{i=1}^{k} p_{i} \ell_{i} \leq \sum_{i=0}^{k-1} E_{i}, \quad \forall k \\
& \sum_{i=1}^{k} p_{i} \ell_{i} \geq\left(\sum_{i=0}^{k} E_{i}-E_{\text {max }}\right)^{+}, \quad \forall k
\end{aligned}
$$

As the optimization problem in (17) is convex with a strictly concave objective function and convex feasible set, it has a unique solution and hence (11) has a unique optimal solution.

\section{The Optimal Policy}

After a Lagrangian analysis [14], we find the optimal total power in epoch $i$ as

$$
p_{i}^{*}=\mu_{s_{i}}\left[\nu_{i}-\frac{1}{\mu_{s_{i}} h_{s_{i}}}\right]^{+}
$$

where $\nu_{i}$ is the water level in epoch $i$ and $s_{i} \in\{1,2\}$. The solution is found by a directional waterfilling algorithm [7]. The algorithm requires walls at the instants of energy arrivals, with right permeable water taps in each wall which allows at most $E_{\max }$ amount of water to flow, as shown in Fig. 2. First, the taps are kept off and transfer from one epoch to the other is not allowed. Each incoming energy $E_{i}$ is spread in the time interval between energy arrivals. The resulting water levels and $s_{i}$ are found by the greedy waterfilling algorithm described in [11], [12]. When each right permeable tap is turned on, the water levels are compared. If the water level on the right is lower, then the resulting water level is found by allowing energy transfer from the past epochs to the epochs. Optimal power allocation $p_{i}^{*}$ is then calculated by plugging the resulting water levels into (18). Individual power shares are then found via (13). An example run of the algorithm is shown in Fig. 2, for a case of 12 epochs. Five energy arrivals occur during the communication session, in addition to the energy available at time $t=0$. We observe that the water level equalizes in epochs 1-5. No power is transmitted in epoch 7, since $\frac{1}{\mu_{s_{i}} h_{s_{i}}}$ is too high. The energy arriving at the beginning of epoch 6 cannot flow left due to causality constraints, which are ensured by right permeable taps. We observe that the excess energy in epochs 6, 7 and 8 cannot flow right, due to the $E_{\max }$ constraint at the beginning of epoch 9 . 


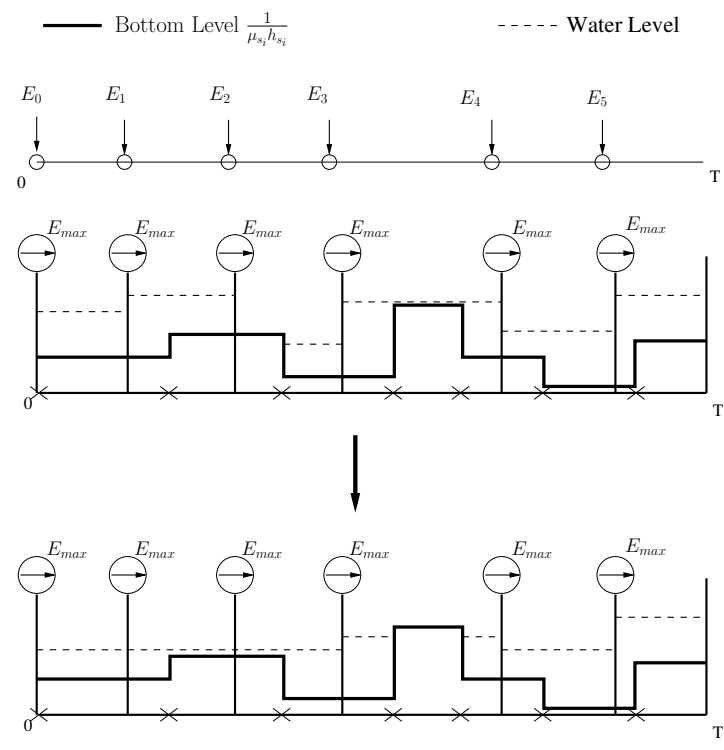

Fig. 2. Directional waterfilling algorithm.

\section{Numerical Results}

We consider a band-limited AWGN broadcast channel with bandwidth $W=1 \mathrm{MHz}$ and noise power spectral density $N_{0}=10^{-19} \mathrm{~W} / \mathrm{Hz}$. The path loss between the transmitter and receiver 1 is $c_{1}=100 \mathrm{~dB}$ and between the transmitter and receiver 2 , is $c_{2}=105 \mathrm{~dB}$. In addition, the channel fading coefficients $h_{1}$ and $h_{2}$ vary during the transmission. We have

$$
\begin{aligned}
& r_{1}=\log _{2}\left(1+\frac{\alpha P}{(1-\alpha) P \mathbf{1}\left(c_{1} h_{1}<c_{2} h_{2}\right)+n_{1}}\right) \text { Mbps } \\
& r_{2}=\log _{2}\left(1+\frac{(1-\alpha) P}{\alpha P \mathbf{1}\left(c_{1} h_{1}>c_{2} h_{2}\right)+n_{2}}\right) \text { Mbps }
\end{aligned}
$$

where $n_{1}=\frac{1}{h_{1}}$ and $n_{2}=\frac{10^{0.5}}{h_{2}}$ and $P$ is in $\mathrm{mW}$. We assume that at times $\mathbf{t}=[0,2,5,8,9,12] \mathrm{s}$, energies with the following amounts are harvested: $\mathbf{E}=[8,3,6,9,8,9] \mathrm{mJ}$. The battery capacity is $E_{\max }=10 \mathrm{~mJ}$. The fading profile, $\mathbf{h}_{i}=\left(h_{1 i}, h_{2 i}\right)$ where $i$ is the time index and both entries are in $\mathrm{dB}$, is defined by $\mathbf{h}_{1}=(7,4), \mathbf{h}_{2}=(7,2), \mathbf{h}_{3}=(2,2), \mathbf{h}_{4}=(-1,3)$, $\mathbf{h}_{5}=(-1,8), \mathbf{h}_{6}=(1,13), \mathbf{h}_{7}=(1,8), \mathbf{h}_{8}=(3,8)$ and $\mathbf{h}_{9}=(5,7)$ at instants $t_{1}^{f}=0 \mathrm{~s}, t_{2}^{f}=1 \mathrm{~s}, t_{3}^{f}=3 \mathrm{~s}, t_{4}^{f}=4 \mathrm{~s}$, $t_{5}^{f}=7 \mathrm{~s}, t_{6}^{f}=8 \mathrm{~s}, t_{7}^{f}=10 \mathrm{~s}, t_{8}^{f}=11 \mathrm{~s}$.

We plot the corresponding maximum departure region for $T=14 \mathrm{~s}$ in Fig. 3. There are four critical points of the maximum departure region, $\mathrm{A}, \mathrm{B}, \mathrm{C}$ and $\mathrm{D}$. At point $\mathrm{A}$, all the power is allocated for the transmission of user 1 and no data is transmitted for user 2; point $\mathrm{D}$ is vice versa. At points $\mathrm{B}$ and $\mathrm{C}$, the priorities of the users are equal, i.e., $\mu=\frac{\mu_{2}}{\mu_{1}}=1$. For the points to the left of $\mathrm{B}, \mu \geq 1$ and for the points to the right of $\mathrm{C}, \mu \leq 1$. The total power allocation at points $\mathrm{A}$ and $\mathrm{D}$ are found by single-user directional water-filling in [7] with the bottom level selected as $\frac{1}{c_{1} h_{1 i}}$ and $\frac{1}{c_{2} h_{2 i}}$, respectively. Moreover, the total power allocation at points $\mathrm{B}$ and $\mathrm{C}$ are identical and found by single-user directional water-filling with the bottom level selected as $\frac{1}{\max \left\{c_{1} h_{1 i}, c_{2} h_{2 i}\right\}}$.

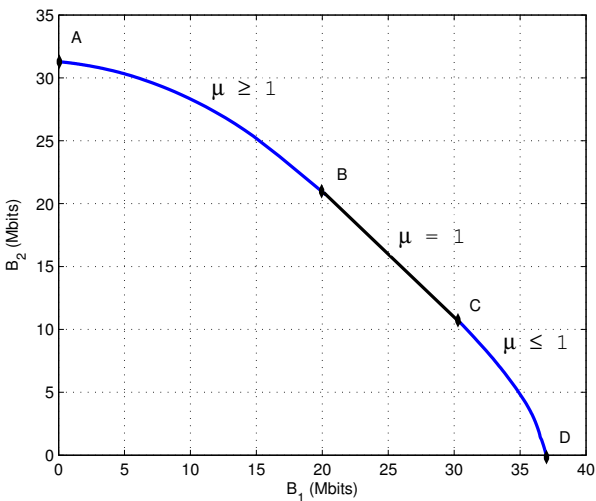

Fig. 3. The maximum departure region for the fading broadcast channel under the given energy arrival and fading profiles at $T=14 \mathrm{~s}$.

\section{CONCLUSIONS}

In this paper, we have considered communication over fading broadcast channels with an energy harvesting rechargeable transmitter. We characterized the region of bit departures by a deadline $T$ in an offline setting. We showed that optimal policies are found by a specific directional waterfilling algorithm.

\section{REFERENCES}

[1] J. Lei, R. Yates, and L. Greenstein, "A generic model for optimizing single-hop transmission policy of replenishable sensors," IEEE Trans. Wireless Commun., vol. 8, pp. 547-551, February 2009.

[2] V. Sharma, U. Mukherji, V. Joseph, and S. Gupta, "Optimal energy management policies for energy harvesting sensor nodes," IEEE Trans. Wireless Commun., vol. 9, pp. 1326-1336, April 2010.

[3] C. Ho and R. Zhang, "Optimal energy allocation for wireless communications powered by energy harvesters," in IEEE ISIT, June 2010.

[4] J. Yang and S. Ulukus, "Transmission completion time minimization in an energy harvesting system," in CISS, March 2010.

[5] J. Yang and S. Ulukus, "Optimal packet scheduling in an energy harvesting communication system," IEEE Trans. Comm., to appear. Also available at [arXiv:1010.1295].

[6] K. Tutuncuoglu and A. Yener, "Optimum transmission policies for battery limited energy harvesting nodes," IEEE Trans. Wireless Comm., submitted, September 2010. Also available at [arXiv:1010.6280].

[7] O. Ozel, K. Tutuncuoglu, J. Yang, S. Ulukus, and A. Yener, "Transmission with energy harvesting nodes in fading wireless channels: Optimal policies," IEEE Journal on Selected Areas in Communications, vol. 29, pp. 1732-1743, September 2011.

[8] J. Yang, O. Ozel, and S. Ulukus, "Broadcasting with an energy harvesting rechargeable transmitter," IEEE Trans. Wireless Comm., submitted, October 2010. Also available at [arXiv:1010.2993].

[9] M. A. Antepli, E. Uysal-Biyikoglu, and H. Erkal, "Optimal packet scheduling on an energy harvesting broadcast link," IEEE Journal on Selected Areas in Communications, vol. 29, pp. 1721-1731, September 2011.

[10] O. Ozel, J. Yang, and S. Ulukus, "Optimal broadcast scheduling for an energy harvesting rechargeable transmitter with a finite capacity battery," IEEE Trans. Wireless Comm., submitted, May 2011.

[11] L. Li and A. Goldsmith, "Capacity and optimal resource allocation for fading broadcast channels-part i," IEEE Trans. Inform. Theory, vol. 47, pp. 1083-1102, March 2001.

[12] D. N. Tse, "Optimal power allocation for parallel gaussian broadcast channels," Technical Report, 1997. Available at www.eecs.berkeley.edu/ dtse/broadcast2.pdf.

[13] T. M. Cover and J. Thomas, Elements of Information Theory. John Wiley and Sons Inc., 2006

[14] O. Ozel, J. Yang, and S. Ulukus, "Optimal transmission schemes for parallel and fading broadcast channels with an energy harvesting rechargeable transmitter," Elsevier Computer Communications, submitted, September 2011. 